\title{
A Natural Language Interface to a Database System
}

Arup Panja, Rami Reddy Nandi Reddy and Sivaji Bandyopadhyay

Computer Science \& Engineering Department, Jadavpur University, Kolkata, India

\begin{abstract}
Two Natural Language Interface to Database (NLIDB) systems have been developed. One is using a Template Grammar based approach and another is using Keyword based approach. Both the systems accept queries in Bengali language. In both the approaches, the user query is analyzed and translated to a SQL statement that is used to retrieve information from the database. The systems have been evaluated based on precision and recall metrics. The NLIDB system based on Keyword based approach has been observed to perform better. The Keyword based approach has been extended further to develop a dialogue based question answering system for Railway information in Telugu language.
\end{abstract}

\section{KEYWORDS}

Natural Language Interface to Database (NLIDB), information extraction, restricted domain question answering, template grammar based approach, keyword based approach, system evaluation

\section{INTRODUCTION}

A natural language interface to a database (NLIDB) (Androutsopoulos et al., 1995) is a system that allows the user to access information stored in a database by typing requests expressed in some natural language (e.g. Bengali).

Reprint requests to: Sivaji Bandyopadhyay, Computer Science \& Engineering Department, Jadavpur University, Kolkata, India; sbandyopadhyay@cse.jdvu.ac.in; sivaji_cse_ju@yahoo.com 
Formal query languages are difficult to learn and master, at least by noncomputer specialists. Graphical interfaces and form-based interfaces are easier to use by occasional users; still, invoking forms, linking frames, selecting restrictions from menus etc. constitute artificial communication larguages that have to be learned and mastered by the end user. In contrast, an ideal NLIDB would allow queries to be formulated in the user's native language. In practice, current NLIDBs can understand only limited subsets of natural language. There are kinds of questions (e.g. questions involving negation, or quantification) that can be easily expressed in natural language, but that seem difficult (or at least tedious) to express using graphical or form-based interfaces. Another advantage of NLIDBs concerns natural language interfaces that support anaphoric and elliptical expressions, where the meaning of each question is complemented by the discourse context.

In this work, we have developed an NLIDB system for an Employee database using Template Grammar based approach (Bandyopadhyay, 2000) and another NLIDB system for a Railway Information System following Keyword based approach. Both these systems are implemented in Bengali, which is an important language in India, the fifth language in the world in terms of speakers and the national language of Bangladesh.

In the Template grammar-based approach, the input query statement is analyzed using Knowledge Base and Template Grammar to produce the corresponding SQL statement. The system is equipped to handle query buffering, materialized view, sorting the output on chosen attribute and also elliptical queries while entering into a dialogue with the user.

In the Keyword based approach, the input query statement is analyzed and based on the keywords in the query an appropriate query frame (Hoojung et al., 2004) is selected. After that, the corresponding procedure for SQL generation is called that uses the semantic information collected during the query analysis. The generated SQL statements are triggered on the database and the retrieved information is shown as a table.

Areas related to NLIDB systems are discussed in Section 2. Section 3 deals with the design of our NLIDB system using Template Grammar based approach while Section 4 details the NLIDB system design using the Keyword based approach. The evaluation has been carried out in Section 5 and the conclusion has been drawn in Section 6. 


\section{RELATED WORKS}

Natural Language Interfaces to database (NLIDB) systems belong to the domain of information extraction. Question Answering (QA) is a type of information extraction; given a collection of documents (such as the WWW) the system should be able to retrieve answers to questions posed in natural language.

Open-domain question answering deals with questions about nearly everything and can only rely on general ontologies. On the other hand, restricted-domain question answering (RDQA) (Diekema et al., 2004, DoanNguyen et al., 2004) deals with questions under a specific domain. If we create such a RDQA interface for structured e.g. relational database, we call it as NLIDB. RDQA has a long history, beginning with systems working over databases (e.g., BASEBALL and LUNAR). One well-known approach for RDQA uses semantic grammars, which build pre-defined patterns of questions for a specific task.

A question answering system that is restricted to the weather forecast domain is described in (Hoojung et al., 2004). In this system, QA engine analyzes natural language questions and translates the questions into SQL statements. These SQL statements are directed to a DBMS to retrieve the answers in the database. Finally, the result from the DBMS is converted to natural language sentences for output.

\section{TEMPLATE GRAMMER BASED APPROACH}

The Employee Database used in the Template Grammar based approach is described in subsection 3.1. The design of the Knowledge Base and Template Grammar rules are mentioned in subsections 3.2 and 3.3 respectively. The architecture of the NLIDB system using Template grammar based approach is described in subsection 3.4. The next subsection 3.5 details how the system handles views, sorting and elliptical queries.

\subsection{Employee Database}

The Employee database used in the Template Grammar Based approach includes the following tables: Employee (Name, Employee_No (primary key), 
Age, Sex, Income, Department_No, Address), Department (Department_No (primary key), Name, Manager_No), Department_Location (Department_No (primary key), Location), Project (Project_No (primary key), Project_Name, Department_No), Works_On (Project_Number (primary key), Employee_No (primary key)) etc.

\subsection{Design of the Knowledge Base}

The words that occur in a database query or in a database command may include words that specify an attribute (i.e., attribute qualifier) or a table (i.e., table qualifier) or the database operation or an aggregate operation or a set operation or a comparison operation or a negation. The knowledge base includes these words and phrases along with the corresponding database objects or operations. The database query may also include values of attributes, both numeric and non-numeric. Numeric attribute values occurring in a query are easy to identify as these are generally followed by attribute qualifiers, but the values of non-numeric attributes may not be followed by attribute qualifiers and hence not easy to identify. A separate knowledge base has been created that includes the values of non-numeric attributes and the corresponding attribute names.

Words for which only the grammatical category is important are stored in a dictionary. We keep a list of Bengali inflections to identify the root words from the surface words in the query.

After analyzing the input query statement into a sequence of tokens, they are to be matched with the template grammar. For this purpose, we have designed a finite state automata corresponding to the Template Grammar and stored its states and state transition characteristics in a State table.

\subsection{Template Grammar}

The input query statement can be considered as a collection of templates in certain order. The parsing process involves identifying the different templates in the input statement.

The mapping from the database templates occurring in the input statement into SQL clauses is done using separate frames for each template. These frames have well defined structures and the values in the slots are filled 
up during the analysis of the input. If some confusion arises during the filling up of a slot, the disambiguation is done using the data dictionary information. Currently, the system is accepting input queries that map to the SQL statement with SELECT, FROM and WHERE clauses. Data retrieval from one or more tables can be specified. Sorting of the records in the output is handled separately. The GROUP BY and HAVING clauses have not been implemented. The different templates and their associated frames are discussed below.

The Select Template can take the following form: (<pronoun | null> (<attribute qualifier> <comma $>$ ) <attribute qualifier> $((<$ conjunction> $<$ attribute qualifier $>$ ) $\mid$ null)). The attribute qualifiers appear together in the input. The pronoun refers to the records in the table. If the input statement is simple then the <pronoun> is absent. The Select Frame is used for generating the identification of the attributes that appears in the SELECT clause and has four fields, namely 'Table_Id', 'Table_Name', 'Attribute_Id' and 'Attribute_Name' that are filled up using the knowledge base.

The From Template has the following form: ((<preposition><determiner> | $<$ determiner $>$ ) ((<value > <table qualifier $>) \mid<$ table qualifier $>)) \mid<$ table qualifier $>$ ). The From Frame is generated from the template and is used for generating the identification of the table in the FROM clause and has two fields, namely 'Table_Id' and 'Table_Name'. The number of frames generated will be same as the number of table qualifiers. The value of the 'Table_Id' field is filled up using the knowledge base.

The Predicates that we are considering at present are of the type: <attribute> <comparison operator> < values / constant > and a number of such predicates can be connected to form the WHERE clause. The Predicate Template can take the following form: ( $<$ non numeric value> <attribute qualifier>) | (<attribute qualifier> <numeric value> <unit> <comparison operator $>$ ) $\mid$ (<numeric value > <unit> <comparison operator $><$ attribute qualifier $>)$ ). The unit qualifies the numeric attribute value. Sometimes, the presence of a non-numeric value signifies a predicate. These Predicate templates are connected to form the Where Template in the following manner: $\{<$ Predicate Template $>$ <conjunction $|<,>|$ null $>$ \}. The corresponding Where Frame is used for generating the WHERE clause and has the following fields, namely 'Table_Id', 'Table_Name', 'Negation', 'Comparison Operator Word', 
'Comparison Operator', 'Value/Constant', 'Attribute_Id' and 'Attribute_Name' that are filled up using the knowledge base.

The input query statement will consist of the Select, From, Where and Database Operation templates in any order because Bengali or any other Indian language has a free word order. In the present work, we have considered that the Database Operation template will occur last in the query statement after the Select template, following the standard word ordering rules of Bengali. The From template and the Where template can occur in the first and second slots of an input query interchangeably.

\subsection{System Architecture}

The architecture of the template grammar based NLIDB system is shown in Figure 1.

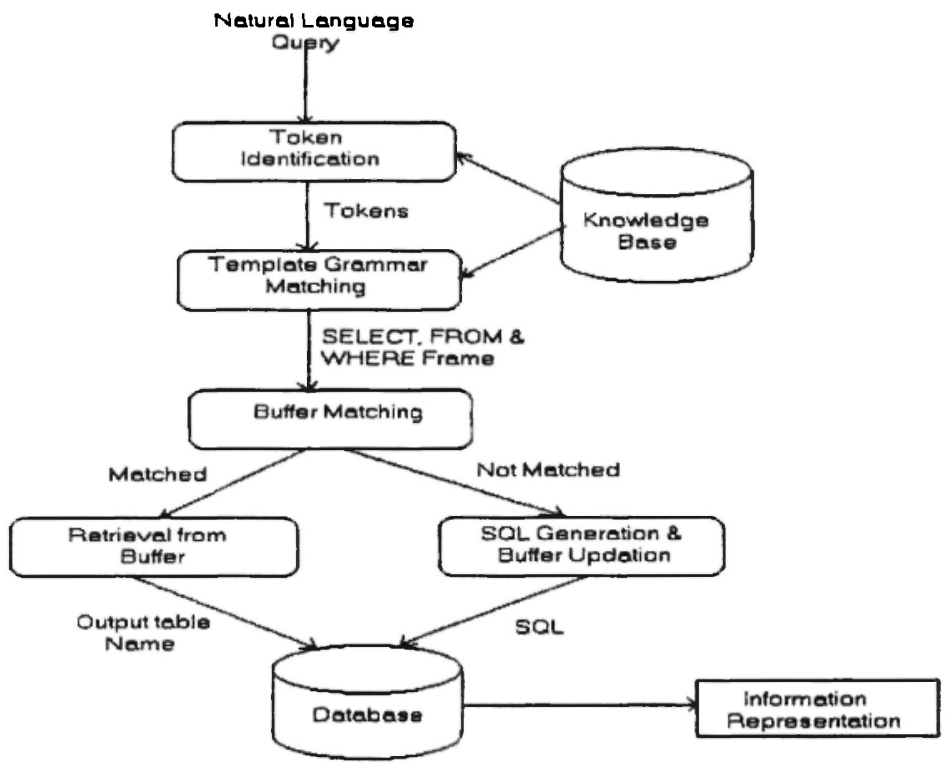

Fig. 1: NLIDB System Architecture (Template Grammar based approach) 
3.4.1 Token identification. In this step, first the input query statement is segmented into a sequence of tokens according to spaces. Next, each token is searched in the knowledge base to determine its type and any semantic information. During this searching, multiwords and inflections are taken into account. The searching process is done simultaneously with the template grammar matching.

3.4.2 Template grammar matching. For matching a part of the input query statement with template grammar we have designed a deterministic finite automata (DFA). The states and the state transition characteristics (i.e., the current state, possible tokens at that state and the next states corresponding to the possible tokens) have been stored in the State Table.

Depending on the current state, the type of the current token in the input query can be one of those stored in the possible token field for the current state in the state table. Accordingly, the word is searched in the knowledge base. If the word is found, then the current state is changed to the state mentioned in the next state field. Otherwise, the query is discarded. Some states in the DFAs are defined as the final state for the corresponding templates. When a template grammar is matched, the slots in the corresponding frame are filled up with values from the tokens.

3.4.3 Buffer matching and $S Q L$ generation. For faster query processing, a Buffer Table has been maintained which stores all the frames of some recent input queries and the corresponding output table names that are kept updated. If all the frames of an input query match in the Buffer Table, then the SQL generation process is bypassed and the output table for the matched query in the Buffer Table is shown to the user. Otherwise, the SQL statement corresponding to the query is generated from the SELECT, FROM and WHERE frames.

In the SELECT frame and the WHERE frame, some attributes may appear which are not attributes of the table in the FROM frame. In that case the FROM frame includes the information of those tables also. Generating FROM clause from the FROM frame is easy when the FROM frame contains only one table. But, if the FROM frame contains more than one table we have to generate the WHERE clause required for joining the tables.

The SELECT clause is generated from the SELECT frame by adding the names of the attributes with comma in between. It could happen that more 
than one attribute from different tables have the same name. To avoid this problem the table name is concatenated with the attribute name.

The WHERE clause may include more than one predicate joined with "and". A predicate may be negated using "not". Each predicate is generated by adding the attribute names, comparison operator, and values.

Finally, the SELECT, FROM and WHERE clauses are concatenated to form the SQL statement. The output table name for the query is entered into the buffer table along with the frames for the query.

3.4.4 Information representation. Once generated, the SQL statement for an input query is triggered on the database and the retrieved information is shown as a table. If the information under some column is in the language different from the query language, translation, or transliteration of data have to be carried out.

\subsection{Views, Sorting and Elliptical Queries}

The system is equipped to handle materialized view, to sort the records in the output on chosen attribute and to handle elliptical queries while entering into a dialog with the user.

In a dialogue system, the first exploratory query is usually followed by many elliptical follow-up queries. The first query is termed as Master Query. For an elliptical follow-up query the FROM frame of the previous query in the dialogue is used for generating the FROM clause. These elliptical queries are expected to refer to the same set of tables as in the Master Query.

The SELECT template usually occurs in the elliptical follow-up queries. A new SELECT frame is created from the SELECT template and the SELECT frame of the previous query is not used in these cases. But if the SELECT template does not occur, the SELECT frame of the previous query is used to generate the SELECT clause.

If the WHERE template does not occur in the follow-up query, then the WHERE frame of the previous query is used to generate the WHERE clause. But if a WHERE template occurs and the preceding part of the input query matches with the pattern like <pronoun><postposition>, then the WHERE frame of the previous query is added with the WHERE frame of the new query to generate the WHERE clause. If a WHERE template occurs but the 
preceding part of the input query does not match the above-mentioned pattern, then a new WHERE frame is created from the WHERE template.

\section{KEYWORD BASED APPROACH}

The Railway Database used in the Keyword based approach is described in subsection 4.1. The design of the Knowledge base is mentioned in subsection 4.2. The architecture of the NLIDB system using Keyword based approach is described in subsection 4.3 .

\subsection{Railway Database}

In a railway information system, database contains information about the arrival / departure time of trains, their fares and their running information etc. The main tables used here are schedule tables for each train, fare tables for special trains like Rajdhani, Shatabdi, etc. that have a different fare structure, Route tables for each route and other tables that include train running frequency details etc. Some temporal tables are maintained in order to check the status of the purchased railway reservation ticket and reservation availability information of a particular journey of a train.

\subsection{Design of the Knowledge Base}

The words that occur in a database query for Railway information system may include words describing Train Name, Station Name, Reservation Class, or Keyword that specifies the topic of the query. Hence, we have maintained a domain dependent ontology in the Knowledge Base in the form of tables.

The Train Name table stores the name of the trains in Bengali and their numbers. In the Station Name table, we have stored the station names in Bengali. In the Reservation Class table, we have stored the name of the reservation classes in Bengali and their aliases (i.e., the short name of the reservation class in English). There is a Bengali Inflection table that can be used in the analysis of the input. A separate Postposition table can be used to identify source, destination, and intermediate station names in the input. In the Keywords table, we have stored the keywords of different types of question and their corresponding query frames. 


\subsection{System Architecture}

The architecture of the keyword based NLIDB system is shown in Figure 2.

4.3.1 Query analyzer. The words in the input query statement are searched in the knowledge base to determine the type and semantic information. During this searching, multiwords and inflections are considered. Some words may not be found in any table. Those words contain no semantic information and are discarded.

Analysis of the entire input query identifies a number of tokens that are used in the SQL generation procedure. Among the tokens, one or more than one token must be the keyword that specifies the question type.

4.3.2 Query frame decision. The scope of the user request can be focused by restricting the query domain and information resource. That is, there are a finite number of expected question topics. Each expected question topic is defined as a single query frame. Some query frame examples for Railway information system are fare of a journey [Fare], arrival [Arr_Time] or departure time [Dep_Time] of a train, trains between important stations [Trains_Imp_Stations], scheduled time [Sched_Time], weekly frequency of a

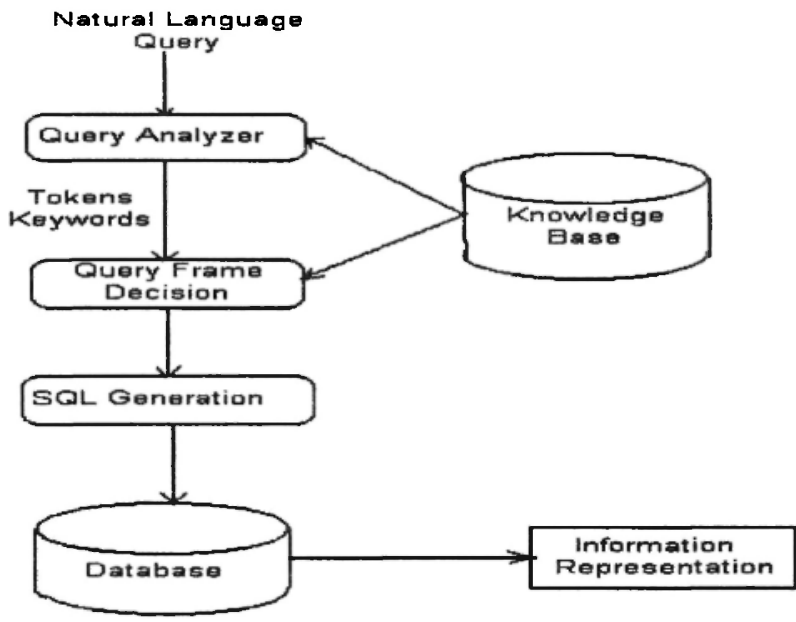

Fig. 2: NLIDB System Architecture (Keyword based approach) 
train [Arr_Frequency/Dep_Frequency], availability of reservation classes in a train [Reservation_Class] and ticket status enquiry [PNR_Enquiry]

During the query analysis, the keywords in the input query are detected. In this step, based on the number of keywords and the type of keywords, we select the appropriate query frame. In some cases, ambiguity will occur in identifying the appropriate query frame. The set of keywords and other tokens in the input query help in disambiguation process.

The query frame-decision process has a great influence on the precision of the system, while there is not much likelihood of errors in other processes, such as generating SQL query from the selected query frame and retrieving database records.

4.3.3 SQL generation. Once a query frame is selected for a question, the corresponding procedure for SQL generation is called. Let us consider that based on the keyword in a query the [Fare] query frame has been selected. Next, the SQL generation procedure for generating the SQL statements to retrieve the fare information is called. The procedure considers that the user will provide the train name and reservation class in the query. It also considers that the user may provide either the source and destination stations of journey or the distance of journey. Each token generated in the query analysis step are checked and the slots Train_Name, Train_No, Reservation_Classes, Source_Station, Destination_Station and Distance are filled with appropriate values. If the source and destination stations are mentioned then it generates two SQL statements for calculating the distance of these stations from the originating station. Then the difference of these distances, i.e., the distance between the source and the destination stations, is calculated. If the distance of journey is provided rather than the source and destination stations the above part of processing is avoided. Next, the final SQL statement is generated which finds out the fare for that distance in the specified reservation classes.

4.3.4 Information presentation. Once generated, the SQL statement for an input query statement is triggered on the database and the retrieved information is shown as a table. If the information under some column is in the language different from the query language, then translation or transliteration of data has to be carried out. 


\section{EVALUATION}

We started our work with the Template grammar based approach on the Employee database and found Keyword based approach is not suitable for the Employee database as the queries on Employee database can not be easily classified based on keywords. Railway information database is suitable for Keyword based approach as the questions can be classified based on the keywords.

The NLIDB system that follows the template grammar based approach on Employee Database has been evaluated by giving 50 queries. Among the 50 queries, the system has generated SQL statements for 36 queries. All these 36 queries have shown the correct result i.e., what the user expected. So the precision and recall of the system have been calculated as follows.

$$
\begin{array}{ll}
\text { Precision }= & \begin{array}{l}
\text { Number of correct SQL statements generated) } / \text { (Number of } \\
\text { SQL statements generated) } * 100 .
\end{array} \\
\text { Recall }= & \begin{array}{l}
\text { Number of correct SQL statement generated) } / \\
\text { (Total number of natural Language query given }) * 100
\end{array} \\
\text { Precision }= & (36 / 36) * 100=100 \% . \\
\text { Recall }= & (36 / 50) * 100=72 \% .
\end{array}
$$

The NLIDB system following the keyword based approach on Railway information database has been evaluated by giving 50 queries. Among the 50 queries the system has generated SQL statements for 48 queries. Among the 48 queries the system has shown correct results for 46 queries. So the precision and recall of the system was calculated as follows.

$$
\begin{aligned}
& \text { Precision }=(46 / 48)^{*} 100=95 \% . \\
& \text { Recall }=(46 / 50)^{*} 100=92 \% .
\end{aligned}
$$

In the template grammar based approach once the SQL statement for a natural language query is generated the output is correct. Hence, precision of this system is always 100 percent. So, precision is not a good measure for comparing these two systems. As the recall of the system following Keyword based approach is better, so we come to a conclusion that the Keyword based approach is better than template grammar based approach. 


\section{CONCLUSION}

In the Template grammar-based approach, the user requires training for querying the database. All the words in the query must be found in the knowledge base to determine its type. Hence, the knowledge base becomes large for processing queries; but in the Keyword based approach only the words that contain semantic information need to be found in the database. As there is no question of grammar matching of any part of the query, the user can be less cautious while querying the database.

The NLIDB system following Template grammar based approach on Employee database can be used on other database by changing the knowledge base only. But the NLIDB system following Keyword based approach on Railway information database cannot be used on other database because, after deciding the query frame for a query, the procedure for SQL generation to retrieve information is fully dependent on the current database it considers. An earlier version of the work was carried out for Bengali language, an important language of India and appeared in (Panja \& Bandyopadhyay, 2005). The Keyword based approach has been extended further for developing a dialogue based question answering system for Railway information in Telugu language (Rami et al. 2006a,b), another important Indian language. The dialogue based QA system is now being extended further to include user models as well as to increase the system services.

\section{REFERENCES}

Androutsopoulos I, Ritchie G. D, and Thanisch P. 1995. Natural language interfaces to databases-an introduction. Natural Language Engineering, 1(Part1), 29-81.

Bandyopadhyay Sivaji. 2000. Anaphora/coreference identification and resolution in a cross language database system. In Proceedings of Discourse Anaphora and Reference Resolution Conference (DAARC. 2000), 197-205.

Diekema A.R, Yilmazel O. and Liddy E.D. 2004. Evaluation of restricted domain question-answering systems. In Proceedings of the ACL 2004 Workshop on Question Answering in Restricted Domain, 2-7.

Doan-Nguyen Hai and Leila Kosseim. 2004. The problem of precision in restricted-domain question answering. some proposed methods of improvement. In Proceedings of the ACL 2004 Workshap on Question Answering in Restricted Domain, 8-15. 
Hoojung Chung, Young-In Song, Kyoung-Soo Han, Do-Sang Yoon, JooYoung Lee, Hae-Chang Rim et al. 2004. A practical QA System in restricted domains. In Proceedings of the ACL 2004 Workshop on Question Answering in Restricted Domain, 39-45.

Panja A. and Bandyopadhyay S. 2005. Design and implementation of a natural language interface to a database (Bengali as a case study). In Proceedings of $2^{\text {nd }}$ Indian International Conference on Artificial Intelligence (IICAI-05), 821-39.

Rami Reddy N.R and Bandyopadhyay S. 2006a. Dialogue based question answering system in Telugu. In Proceedings of EACL 2006 Workshop on Multilingual Question Answering. ISBN: 2-9524532-4-1.

Rami Reddy N.R and Bandyopadhyay S. 2006b. Keyword based shallow parsing for a restricted domain QA system in Telugu. In Proceedings of First National Symposium on Modeling and Shallow Parsing of Indian Languages (MSPIL 06), 257-66. 